\title{
Analyse météorologique de l'évolution temporelle du risque d'inondation à Douala (Cameroun)
}

\author{
Rinelle Djiondo Ngongang ${ }^{1}$, Lucie Angennes Djiotang Tchotchou ${ }^{2}$, \\ Boniface Elvis Ossombo', Brice Baudouin Sandjeu Wandjie', \\ André Lenouo $^{1}$ \\ 1. Laboratoire de physique fondamentale, Département de Physique, Faculté \\ des Sciences, Université de Douala, Cameroun \\ 2. Département de Physique, Faculté des Sciences, Université de Yaoundé I, \\ Cameroun
}

drinelle7@yahoo.fr

lenouo@yahoo.fr

\section{Résumé}

Cet article analyse l'évolution des inondations dans la ville de Douala à partir des méthodes permettant de calculer une valeur de débit maximum à l'aide des données météorologiques sur une période de 35 ans (1985-2019). Une base de données sur les inondations créée à l'aide d'informations issues de la presse montre que Douala est fréquemment inondée durant la saison des pluies par des systèmes précipitants en $V$ associés à la mousson. On observe ainsi une augmentation du nombre d'inondations rapportées depuis les années 2010, qui semble corrélée avec l'évolution de l'emprise urbaine de la ville. Les zones urbaines inondées de façon récurrente sont le plus souvent les quartiers " informels » construits directement en zones inondables et dont l'accroissement est important depuis les années 1990. L'étude des inondations du 2 et 3 août 2000 à Douala indique qu'on avait une situation synoptique propice à de fortes précipitations. On peut par l'exemple noter que l'humidité relative était presque de $99 \%$, ce qui implique que l'air est saturé en eau avec es eaux pluviales sont les eaux issues des précipitations atmosphériques qui s'écoulent sur une surface. L'eau de ruissellement rejoint ensuite le réseau d'assainissement. Lorsqu'elle a ruisselé sur une surface non contaminée, elle peut être collectée pour être utilisée par exemple pour l'arrosage et les chasses d'eau des cabinets d'aisance. Lorsqu'elle n'arrive plus à s'infiltrer dans le sol et que le réseau hydraulique sature, on assiste le plus souvent à des inondations lors d'épisodes de précipitations à la fois très intenses et très étendues (Zizkova, 2004). L'imperméabilisation du sol (bâtiments, voiries, parkings, etc.) limite l'infiltration des eaux de pluies et accentue le ruissellement. Les inondations par ruissellement en secteur urbain sont celles qui se produisent par écoulement dans les rues de volumes d'eau ruisselée sur le site ou à proximité et qui ne sont pas absorbés par le réseau d'assainissement superficiel et souterrain.

Les inondations représentent la principale cause des catastrophes naturelles dans le monde (Bertoni, 2006; De Groeve et al., 2006 ; Paprotny et al., 2018) et l'Afrique ne fait pas exception. De plus, l'Afrique subsaharienne est considérée comme la sous-région la plus pauvre du continent et de la planète (Giec, 2007). Face aux changements climatiques, les populations sont très vulnérables. Divers facteurs participent à leur vulnérabilité. Ils sont liés à l'environnement urbain (densité, croissance démographique, urbanisation incontrôlée galopante, imperméabilisation du sol, etc.) et à sa gestion, à la pauvreté et à tous ses corollaires, ainsi qu'à la sensibilité aux catastrophes naturelles, notamment aux inondations (Wallez, 2010). S'interroger sur la capacité des acteurs à gérer et surtout à s'adapter à cette situation est primordial. Dans le contexte des changements climatiques, il est nécessaire d'anticiper les événements avant qu'ils ne deviennent des catastrophes. Cela passe par la compréhension et une meilleure connaissance desdits événements.

Dans ce travail, on s'est intéressé aux inondations dans le bassin de Douala (Cameroun). Les inondations sont des événements soudains et ponctuels. Leur étude requiert des données sur de longues périodes. Or, celles-ci sont actuellement limitées dans cette région (Giec, 2007). De plus, le Giec souligne 
une chute de température. L'intérêt de cette étude est de réduire la vulnérabilité en intégrant les populations et les décideurs au cœur des stratégies de développement et de gestion urbaine des risques liés aux aléas climatiques.

\section{Abstract}

\section{Meteorological analysis of the temporal evolution of the flood risk in Douala (Cameroon)}

This article analyzes the temporal evolution of flooding in the urban area of Douala using methods allowing to compute a maximum flow value using meteorological data over a period of 35 years (1985-2019). A flood database is created using information from the press showing that Douala is frequently flooded during the rainy season by $\mathrm{V}$-shaped precipitating systems associated to the monsoon. We can thus observe an increase in the number of floods reported since the 2010s, which seems to be correlated with the evolution of the city's urban footprint. The recurrently flooded urban areas are most often informal neighbourhoods built directly in flood-prone areas and whose increase has been significant since the 1990s. The case study of the floods of August 2 and 3, 2000 in Douala indicates that there was a synoptic situation conducive to heavy rainfall. On the other hand, relative humidity is almost $99 \%$ which requires the air to be saturated with water with a drop in temperature, which will cause a change of state. The purpose of this study is to reduce vulnerability by integrating populations and decision-makers at the heart of development strategies and urban risk management related to climatic hazards. l'augmentation probable des « risques de phénomènes météorologiques extrêmes » en lien avec les changements climatiques, à savoir l'intensité et la fréquence et, de fait, la gravité des sécheresses, vagues de chaleurs et inondations devrait s'amplifier.

Le manque d'informations hydrologiques suffisantes et fiables est un obstacle majeur à la planification des ressources en eau et à leur gestion en Afrique. Par conséquent, Karamage et al. (2018) ont examiné la relation entre les précipitations et le ruissellement à trois échelles spatiales, comprenant l'ensemble du continent, 25 grands bassins et 55 pays. À cette fin, le coefficient de ruissellement a été estimé à l'aide des données de ruissellement mensuelles à long terme calculées à partir des enregistrements de débit du Global Runoff Data Center (GRDC) et des ensembles de données de précipitations du Global Precipitation Climatology Center (GPCC) pour la période allant de 1901 à 2017. Plusieurs modèles ont été utilisés pour étudier les différents bassins en Afrique (Wallez, 2010 ; Jung et al., 2017 ; Weerasinghe et al., 2020), mais le lien entre les précipitations et les inondations à travers le ruissellement peut permettre de documenter ce problème dans la zone côtière du golfe de Guinée. Dans la suite, nous allons présenter la zone d'étude, les méthodes pour déterminer du ruissellement et sa corrélation avec les grandeurs météorologiques (la précipitation, la température au sol et l'évapotranspiration). Il faut noter que l'évapotranspiration se définit comme étant la quantité d'eau qui s'évapore par le sol, les nappes liquides et la transpiration des végétaux, donc on peut dire que c'est une combinaison de deux processus, l'évaporation et la transpiration (Jin et Shepherd, 2005). Enfin, nous évaluerons la période de retour des inondations dans le bassin de Douala après l'étude des inondations des 2 et 3 août 2000 .

\section{Domaine d'étude, données et méthodes}

\section{Domaine d'étude}

Douala est située sur la côte du golfe de Guinée au pied de la chaîne de montagnes du Cameroun, du côté de la mer (figure 1). Capitale économique du Cameroun, elle est à 9,7 de longitude est et $4,0^{\circ}$ de latitude nord. Son climat est très chaud et les pluies sont intermittentes (Sighomnou et al., 1993 ; Lenouo et al., 2009 ; Tanessong et al., 2017). On note sur la carte topographique que l'altitude maximale est d'environ 100 mètres, minimale de 7 mètres avec une moyenne de 17 mètres. Bruckmann et al. (2019) observent que ce ne sont pas les zones les plus basses qui sont les plus fréquemment inondées, à l'exception du quartier de Bonabéri qui est densément drainé et fortement peuplé. Celui-ci est construit en rive droite du Wouri, sur une zone de mangrove, en extension $\mathrm{du}$ tissu industriel. L'importance de la densité du réseau hydrographique dans l'occurrence des inondations se retrouve également dans les quartiers de BP Cité, Bépanda, New-Bell et Maképé Missoké.

Douala a des précipitations importantes la plupart des mois, avec une saison sèche courte (figure 2). D'après

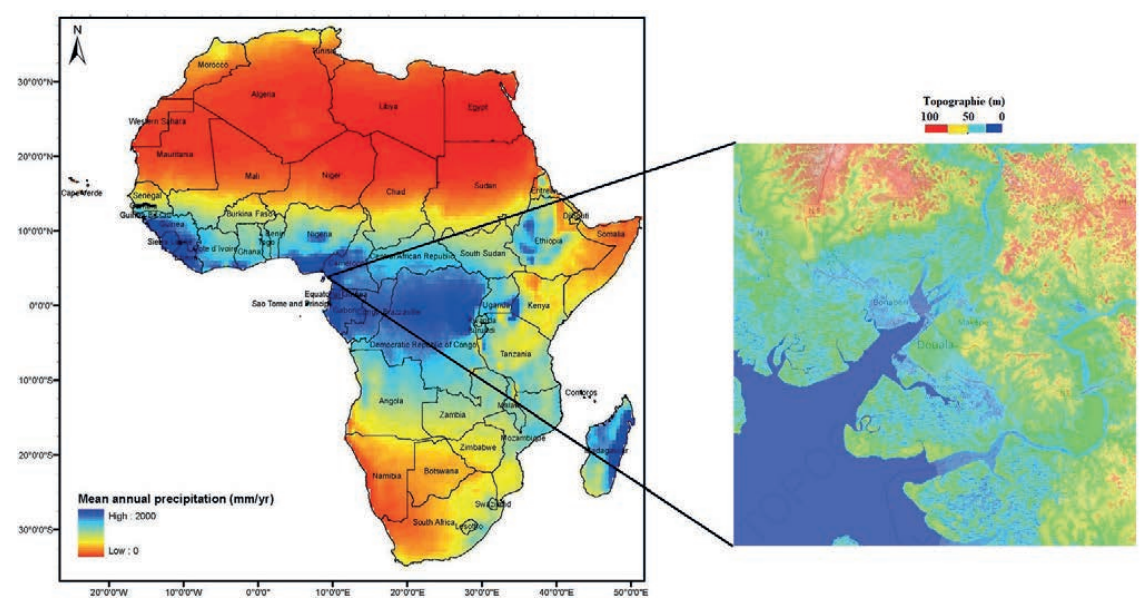

Figure 1. Carte du continent africain avec les noms des pays et les régimes de précipitations. Source : ERA-Interim corrigé avec GPCP v2.1 ; période : 1979-2010. Voir Masih et al. (2014) pour une explication détaillée. En zoom, localisation de Douala, des zones urbaines, des principaux bassins versant et de la topographie. 


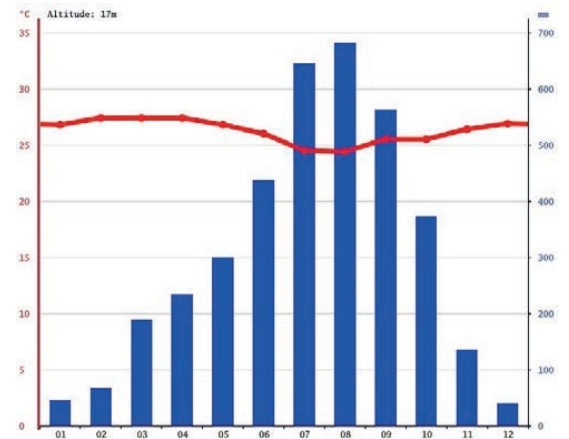

Figure 2. Diagramme ombrothermique de Douala avec un climat dominant de type tropical. Les précipitations sont importantes une grande partie de l'année, avec une saison sèche courte et une température moyenne de $26,2^{\circ} \mathrm{C}$. Source : climate-data.org.

la classification de Köppen et Geiger ${ }^{1}$, le climat y est classé Am, soit un climat de mousson. En moyenne, la température à Douala est de $26,2{ }^{\circ} \mathrm{C}$. La moyenne des précipitations annuelles atteint $3702 \mathrm{~mm}$; avec $39 \mathrm{~mm}$ de précipitation, le mois de décembre est le plus sec de l'année. Le mois d'août, avec une moyenne de $681 \mathrm{~mm}$, affiche les précipitations les plus importantes. Le mois de février est le plus chaud de l'année, avec $27,4^{\circ} \mathrm{C}$, alors qu'au mois d'août la température moyenne est de $24,4^{\circ} \mathrm{C}$. Août est de ce fait le mois le plus froid de l'année. Une variation de $3,0{ }^{\circ} \mathrm{C}$ est donc enregistrée sur l'année.

Les travaux d'Amanejieu (2019) révèlent une augmentation de la surface urbaine de Douala de plus de 140000 hectares entre 1986 et 2017, soit environ 4502 ha par an et 9 ha environ par semaine, avec une extension de la ville vers les zones de basses altitudes potentiellement inondables. En 31 ans, 2830 ha de zones basses ont ainsi été urbanisées sur des remblais. Cette conquête des zones basses a conduit à des inondations dont les premiers cas ont été enregistrés à partir de 1984. De 1984 à 2018, plusieurs inondations ont été recensées et ont occasionné 49 pertes en vies humaines, 71 blessés, 90923 sinistrés et 547 relogés (Amanejieu, 2019). On est passé de 8 inondations pour 8 décès et 8023 sinistrés entre 19842000, à 26 inondations pour 41 décès et 82900 sinistrés entre 2001-2018. Pour faire face à ces inondations de plus en plus fréquentes, les autorités ont opté pour une nouvelle forme de gouvernance urbaine depuis 1993 : le drainage et le délogement de la

1. https://www.britannica.com/science/Koppenclimate-classification population des zones à risques. De 1993 à 2018, 89060 personnes ont été déplacées en 16 vagues (Amanejieu, 2019).

\section{Les données utilisées}

Les données de réanalyse utilisées dans ce travail sont issues du Centre européen pour les prévisions météorologiques à moyen terme (CEPMMT). Ce centre met à disposition les sorties d'un modèle global atmosphérique (IFS) dont la particularité est d'assimiler régulièrement des données mesurées, récoltées sur l'ensemble du globe à heures fixes (température, humidité, pression, vent, nébulosité... issues de mesures in situ, de radiosondages, de données satellitaires, etc.) de façon à ce que les prévisions à moyen terme découlent d'un état initial le plus proche possible de la réalité. Les résultats de l'assimilation de données constituent les analyses opérationnelles. Les réanalyses atmosphériques globales du CEPMMT, ERA 40 (Uppala et al., 2005) et ERA-Interim (Simmons et al., 2006), sont réalisées sur le même principe que les analyses opérationnelles. ERA-Interim ont les données disponibles de 1979 à 2019 avec une résolution spatiale de $0,75^{\circ} \times 0,75^{\circ}$.

Les données in situ à Douala sont issues de Meteoblue. Elles proviennent de nombreux centres météorologiques nationaux, ainsi que d'autres sources complémentaires (Direction de la météorologie nationale (DMN) du Cameroun et de l'Institut de recherche et de développement (IRD)). Les conditions initiales sont habituellement déterminées grâce aux mesures et aux observations qui recouvrent les lieux et les variables météorologiques les plus importantes, mais elles décrivent uniquement une fraction de la surface de la Terre et des processus météorologiques. Ces mesures sont ensuite incorporées dans les simulations du modèle (assimilation de données) pour déterminer l'état du temps sur l'ensemble du globe. Après l'utilisation du modèle, les données de sortie peuvent être validées et corrigées grâce à des données de mesure et d'observation, en utilisant des techniques de post-traitement, comme la réduction d'échelle, les statistiques, l'apprentissage automatique (machine learning) et les prévisions immédiates (nowcasting). En général, Meteoblue offre par défaut l'ensemble de données ayant la plus grande précision (pour les données disponibles sur le site web de Meteoblue, l'API ou History+). Néanmoins, à des fins professionnelles, le choix judicieux des données météorologiques est crucial pour la réussite du projet. Pour faire ce choix avec succès, de nombreux aspects doivent être pris en compte. Toutes les données peuvent être téléchargées pour les lieux sélectionnés pour une période de plus de 30 ans à partir de 1985. Dans ce travail, nous avons sélectionné les données journalières sur la période 1985-2019 (35 ans), ceci afin d'avoir une série suffisante pour les calculs statistiques.

\section{Formules statistiques}

Les outils statistiques utilisés dans le cadre de ce travail sont : la moyenne, la variance, la tendance, la corrélation et la période de retour. Les termes période de retour (PR) et niveau de retour (NR) sont utilisés pour décrire la probabilité d'occurrence des événements et sont déduites du type de modèle d'ajustement aux données empiriques (Cooley, 2013). La PR peut être définie comme la fréquence d'occurrence d'un événement d'une certaine intensité, tandis que le NR est l'intensité ou le quantile d'un événement. Les ambiguïtés liées à la notion de temps de retour viennent $\mathrm{du}$ fait que, faute d'une connaissance suffisante de la physique des processus météorologiques et hydrologiques, l'estimation des probabilités s'est jusqu'à présent essentiellement appuyée sur l'étude de chroniques d'observations.

Dans cadre de ce travail, les NR et $\mathrm{PR}$ des inondations de juin à août sont estimés à l'aide de la relation suivante (Chamani et al., 2019) :

$$
\mathrm{NR}=\mu+\frac{\sigma}{\xi}\left[1-(\lambda \cdot \mathrm{PR})^{-\xi}\right]
$$

où $\lambda$ est le nombre moyen de dépassements du seuil par an ; $\lambda$ informe sur la fréquence des événements extrêmes et est égal au nombre de dépassements de seuil divisé par le nombre d'enregistrement. Par ailleurs, $\mu$ est le seuil sélectionné. Cette série de distribution est caractérisée par deux paramètres : $\sigma$ le paramètre d'échelle et $\xi$ le paramètre de la forme.

\section{Résultats}

\section{Variabilité de quelques paramètres météorologiques}

La figure 3 montre la variabilité de quelques paramètres météorologiques à Douala durant la période 1985-2019. Nous observons un pic de précipitations en juillet, ce qui est dû à la saison 
(a)
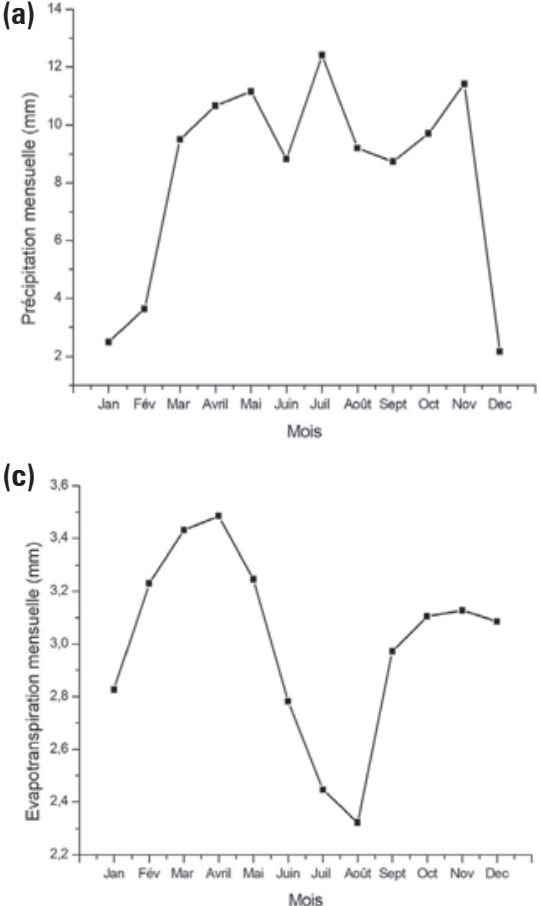

(b)

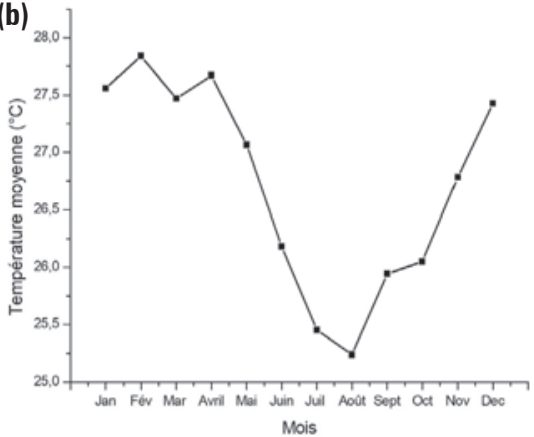

(d)

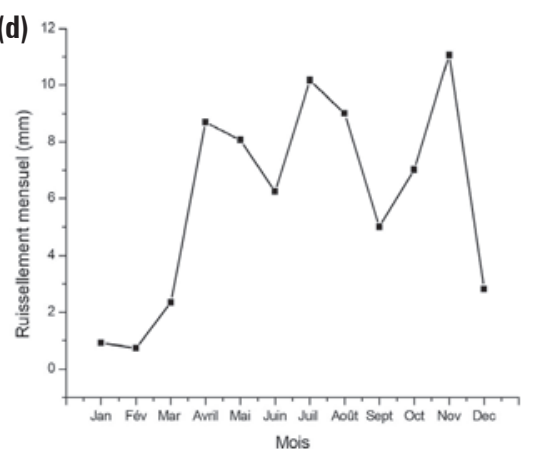

Figure 3. Variations mensuelles moyennes : (a) des cumuls de précipitations (en $\mathrm{mm} / \mathrm{mois}$ ), (b) de la température (en ${ }^{\circ} \mathrm{C}$ ), (c) de l'évapotranspiration (en $\mathrm{mm} / \mathrm{mois}$ ) et (d) du ruissellement (en $\mathrm{mm} / \mathrm{mois}$ ) à Douala (environ 210 km²) durant la période 1985-2019.

pluvieuse (juin, juillet, août) qui enregistre près de $80 \%$ des pluies annuelles (Tsalefac et al., 2003). Durant la même période, les vents d'ouest de l'océan Atlantique sont fortement déviés vers la côte camerounaise (Lenouo et al., 2009), cela permet également d'expliquer les faibles températures en juillet et août. Le mois de novembre enregistre la quantité de précipitations la plus élevée après juillet, ceci est un effet de la variabilité climatique dans la ville de Douala, car c'est un mois de transition entre deux saisons (Lenouo et al., 2009). Nous pouvons constater que l'évapotranspiration déterminée par la méthode de Penman-Monteith (Wandjie et al., 2020) varie de la même façon que la température (figure $3 \mathrm{~b}$, c). En novembre et en juillet, les ruissellements sont les plus élevés durant l'année, ces deux mois correspondant également aux mois de précipitations les plus élevés, ce qui n'est pas le cas avec les autres paramètres.

\section{Variabilité mensuelle des ruissellements}

Nous avons précédemment décrit plusieurs méthodes pour calculer les ruissellements (voir encadré). La figure 4 donne la représentation des trois méthodes, les valeurs des ruissellements sont moyennées sur chaque mois de l'année. Les ruissellements observés sont obtenus déjà à partir de

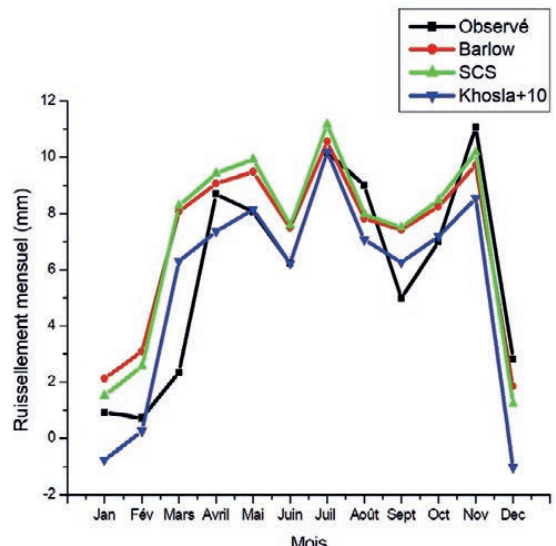

Figure 4. Variations mensuelles des différents ruissellements $(\mathrm{mm})$ obtenues à partir des valeurs observées (les données in situ à partir de Meteoblue) et des méthodes de Khosha modifiée, Barlow et SCS durant la période 19852019.

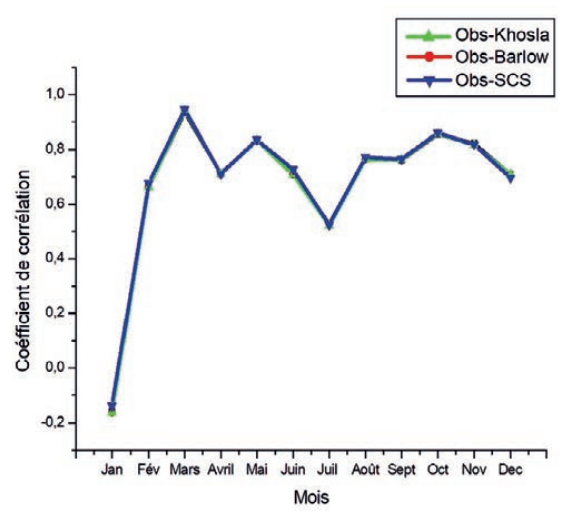

Figure 5. Corrélation entre différents ruissellements mensuels à Douala durant la période 1985-2019. l'hydrogramme des ruissellements tel que le recommande l'OMM $\left(\mathrm{n}^{\circ} 1072\right.$ du « Manuel sur la prévision et l'annonce des crues $»$.

Nous remarquons une bonne corrélation entre les ruissellements observés et ceux obtenus à l'aide des méthodes de Khosla, Barlow et SCS. On rappelle que la méthode de Barlow est valide pour les surfaces inférieures à $130 \mathrm{~km}^{2}$, SCS (Soil Conservation Services) est la méthode de calcul des ruissellements journaliers (Monfet, 1979), alors que la méthode de Khosla est en fonction de la température. On a des valeurs négatives dans la zone d'étude, parce qu'elle a été conçue pour le nord de l'Inde dépendant des paramètres climatiques (Water Supply Engineering Punmia). La figure 4 indique aussi que la méthode de Barlow et celle de SCS ont une différence de moins de $0,5 \mathrm{~mm}$ et de $1 \mathrm{~mm}$ avec le ruissellement observé ; par exemple, en novembre, le ruissellement le plus élevé est celui des valeurs observées, puis celui de SCS. Les corrélations nous aideront à identifier la méthode qui réalise le meilleur ajustement.

\section{Corrélation entre le ruissellement observé et les ruissellements estimés}

La corrélation entre le ruissellement observé (données obtenues de Meteoblue) et celui obtenu par les autres méthodes (Khosla, Barlow et SCS) varient de 0,5 à 0,85 de février à décembre, ce qui montre une bonne corrélation entre ces méthodes. En janvier, nous avons un coefficient de corrélation négatif, qui peut être dû à la saison et au lieu. La méthode SCS est adéquate au calcul des ruissellements journaliers ; elle a une très forte corrélation avec les autres méthodes, ce qui montre qu'elle peut être applicable partout. Il y a une parfaite corrélation entres les méthodes de Barlow et de Khosla.

\section{Corrélation entre le ruissellement et les paramètres météorologiques}

La figure 6 montre une forte corrélation entre les précipitations et les ruissellements de février à décembre et une très faible corrélation en janvier ; ceci permet de dire que les ruissellements dépendent principalement des précipitations. Cette figure montre aussi 
que, durant les mois de janvier, mars, septembre, octobre, novembre et décembre, la corrélation est très faible entre la température et le ruissellement, ceci peut s'expliquer par le fait que les ruissellements dépendent aussi des régions et des saisons. Par contre, en février, avril, mai, juin, juillet et août, le coefficient de corrélation varie de $-0,65$ à $-0,5$, ce qui nous permet de dire que lorsque la température augmente les ruissellements diminuent et vice versa. Douala étant une zone urbaine, l'expansion urbaine favorise l'îlot de chaleur urbain d'environ $0,34{ }^{\circ} \mathrm{C}$, ce qui augmente la température de l'air de $1,6{ }^{\circ} \mathrm{C}$ (Jones et al., 1990). Cette augmentation modifie la couverture végétale en réduisant la quantité d'eau

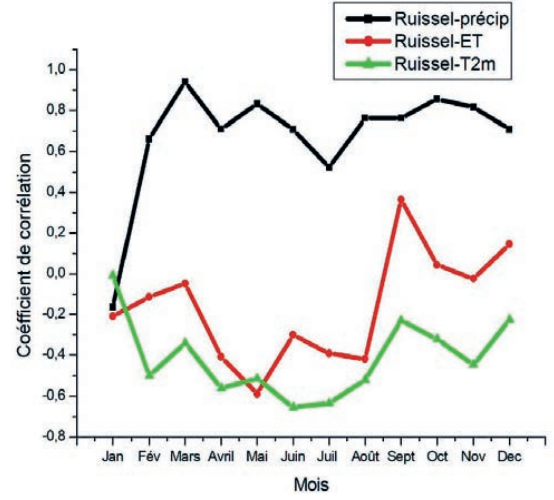

Figure 6. Corrélation entre les ruissellements et les précipitations, les ruissellements et la température à 2 mètres du sol, et les ruissellements et l'évapotranspiration à partir des moyennes mensuelles au cours de la période 1985-2019.

\section{Méthodes de détermination des ruissellements}

\section{Formule de Khosla}

En 1949, Khosla a développé une méthode appliquée au nord de l'Inde pour estimer le ruissellement :

$$
Q=P-\frac{T-32}{3,37}
$$

$Q$ est le ruissellement en $\mathrm{mm}, P$ la précipitation en $\mathrm{mm}$ et $T$ la température en Farenheit.

\section{Formule de Barlow}

La formule de Barlow est dérivée de la méthode rationnelle, le coefficient de ruissellement dépend des facteurs qui affectent le ruissellement ; elle est applicable sur les surfaces inférieures à 130 km². La méthode rationnelle est l'une des plus anciennes. Elle remonte au milieu du XIX ${ }^{\mathrm{e}}$ siècle (Mulvaney, 1851). C'est à Kuichling (1889) qu'est généralement attribuée la paternité du concept, qui a survécu jusqu'à nous à peu près dans sa forme originelle. Elle est considérée valide pour les superficies de moins de 800 ha. Elle suppose que le débit maximum est obtenu lorsque toute la superficie du bassin-versant contribue au ruissellement à l'exutoire avec la plus grande intensité moyenne de précipitation. Cette plus grande intensité correspond à la plus grande précipitation pour la durée du temps de concentration. Elle est fondée sur la proportionnalité et la linéarité de la transformation pluie-débit, exprimées par la relation:

$$
Q=\text { CiA }
$$

$Q$ désigne le débit de pointe à l'exutoire (en $\mathrm{mm}), \mathrm{C}$ le coefficient de ruissellement sur le bassin versant, $i$ l'intensité moyenne de la pluie (en $\mathrm{mm} / \mathrm{h}$ ) pour une durée égale au temps de concentration et $A$ superficie du bassin versant (en ha). La notion de coefficient de ruissellement reste néanmoins assez délicate d'emploi, car C est loin d'être constant et varie, pour un même site, avec la nature, le volume et l'intensité de la pluie, ainsi qu'avec les divers types de surfaces (Pratt et al., 1984). La détermination précise de la valeur de $C$ et de l'intensité moyenne de la pluie reste donc difficile (Bruckmann et al., 2019) et passe par des mesures sur site. À Douala, la valeur moyenne de $C$ est d'environ

0,228 et, pour les précipitations, on va considérer les intensités maximales, car elles sont responsables des inondations observées dans cette zone (Bruckmann et al., 2019).

\section{Méthode du Soil Conservation Service (SCS)}

Le modèle du SCS s'appuie sur le concept que la précipitation totale tombant sur un bassin peut être séparée en trois composantes : le ruissellement direct $(Q)$, la rétention qui se produit dans le bassin $(F)$ et les pertes initiales $\left(I_{\mathrm{a}}\right)$. La relation entre la précipitation $(P)$ et les trois composantes est la suivante :

$$
\frac{F}{S}=\frac{Q}{P-I_{a}}
$$

où $S$ représente la rétention potentielle maximale.

La rétention se produisant réellement se définit avec la relation suivante :

$$
F=\left(P-I_{\mathrm{a}}\right)-Q
$$

Substituant la relation (2) dans (1), on obtient :

$$
\frac{\left(P-I_{\mathrm{a}}\right)-Q}{S}=\frac{Q}{P-I_{\mathrm{a}}}
$$

En réarrangeant la relation (3), on trouve finalement:

$$
Q=\frac{\left(P-I_{\mathrm{a}}\right)^{2}}{P-I_{\mathrm{a}}+S}
$$

$\operatorname{avec} P \geq I_{\mathrm{a}}$.

Soulignons que tous ces paramètres sont exprimés en unités identiques (en millimètres pour le système $\mathrm{SI}$ ou en pouces pour le système impérial). Cette équation contient une quantité connue $P$ et deux inconnues $\left(I_{\mathrm{a}}\right.$ et $S)$. On pose ensuite, en se basant sur les résultats de simulations, que la perte initiale $I=0,2 \times S$. Ceci donne donc en transposant dans l'équation (4) :

$$
Q=\frac{(P-0,2 S)^{2}}{P+0,8 S}
$$

Cette relation est l'équation de base permettant de calculer le ruissellement $Q$ à partir des précipitations $P$. disponible pour l'évapotranspiration ; ceci entraîne la baisse de l'humidité relative (Lyn et al., 2008). En mai, lorsque les ruissellements augmentent, l'évapotranspiration diminue.

\section{Étude de cas : les inondations du 2 et 3 août 2000 à Douala}

Les inondations des 2 et 3 août 2000 semblent être les plus meurtrières en termes de vies humaines, même si celles du 21 août 2020 étaient parmi les plus spectaculaires, mais sans aucune perte en vies humaines.

En observant l'alternance de la température et du vent à minuit le 2 août, on constate que la température de l'air était basse et diminue continuellement vers Douala au fur et à mesure que le vent se déplaçait de la mer vers la côte. Et à la même heure, l'humidité relative était presque de $99 \%$, ce qui implique que l'air est saturé en eau. Par la suite (à $6 \mathrm{~h}$ ), nous constatons que la température est plus élevée de la mer vers la côte suivant la direction du vent, mais moyenne vers Douala ; l'humidité relative baisse légèrement, ce qui traduit une baisse négligeable des précipitations. À $12 \mathrm{~h}$, on constate une baisse progressive de l'humidité relative et de la température en direction de la côte ouest, d'où la présence des fortes pluies. En soirée (18 h), la température baisse en direction de la côte de Douala et l'humidité relative est très élevée aux environs de Douala; on peut dire que le processus de condensation de la vapeur d'eau a repris son cours, d'où la forte précipitation.

Du 2 au 3 août à minuit, il y a variation de température à la hausse vers Douala, la teneur en eau est saturée, ce qui pourra entraîner une grande pluie. Au petit matin, l'humidité était à $100 \%$, avec une chute de température de la mer vers la côte dans la direction du vent. À partir de $18 \mathrm{~h}$, la température diminue progressivement, le vent se déplace de la mer vers la côte ; on a une teneur en eau saturante dans toute la zone et majoritaire au niveau de la côte. Nous pouvons donc dire que les inondations du 2 au 3 août 2000 dans la ville de Douala étaient dues à une variation brusque de l'humidité relative et une chute constante de la température (figures 7 et 8). Cela pourrait également être causé par la géomorphologie de Douala, sa position par rapport au niveau de la mer et la faible capacité d'infiltration des sols 
t2m_vent_oh_2

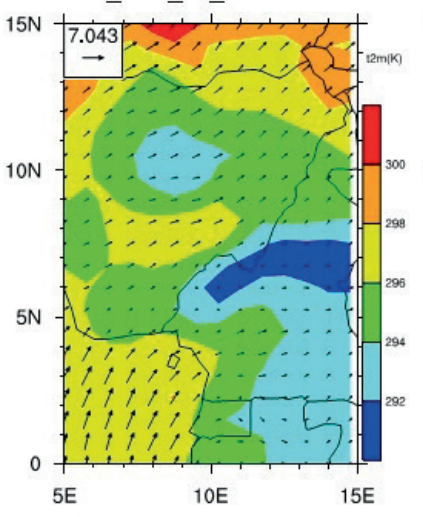

t2m_vent_12h_2

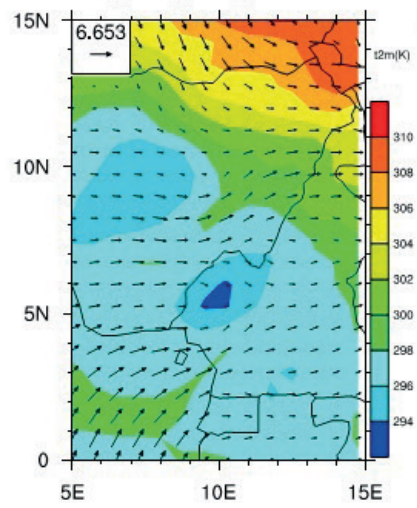

t2m_vent_6h_2

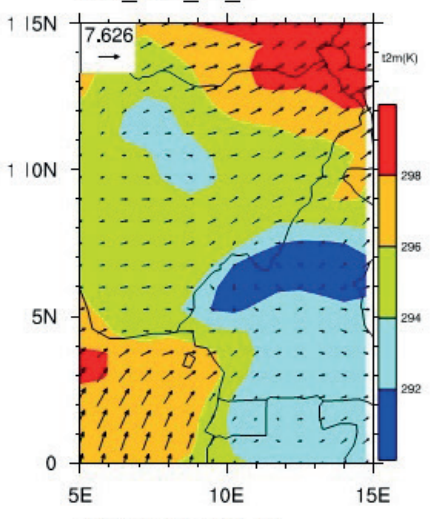

t2m_vent_18h_2

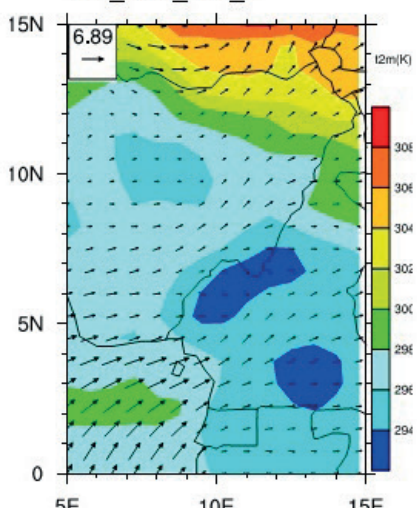

t2m vent oh
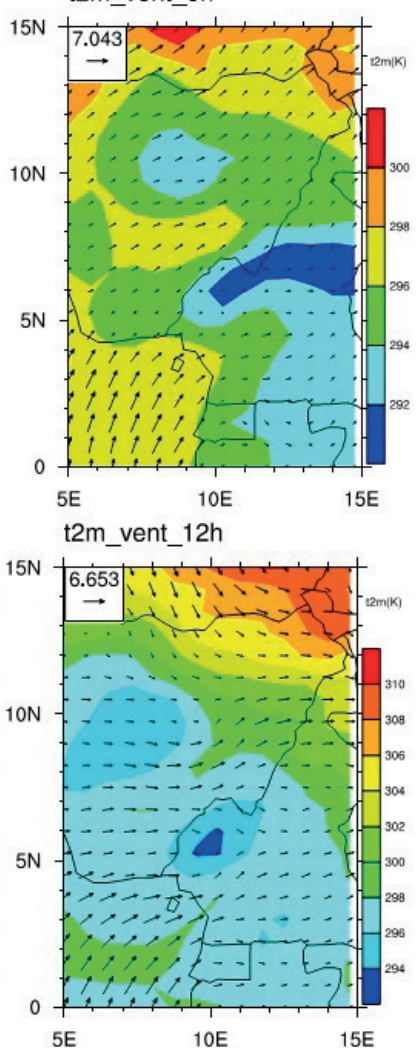

t2m_vent_6h

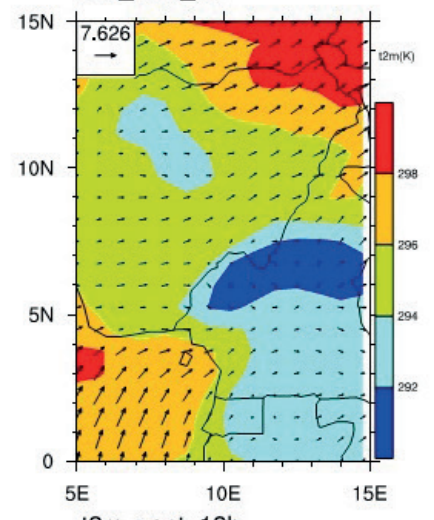

t2m_vent_18h

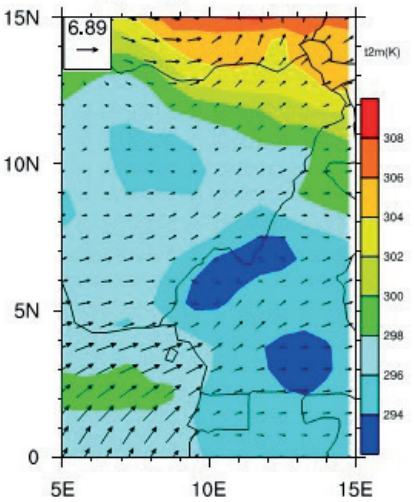

Figure 7. Variation horizontale de température à 2 mètres (en K) et du vent à 850 hPa toutes les 6 heures, du 2 août 2000 (à 0, 6, 12 et 18 h, les 4 panels de gauche) au 3 août 2000 (à 0, 6, 12 et 18 h, les 4 panels de droite).

en saisons pluvieuses, ce qui a été la cause des pertes en vies humaines et des dégâts matériels. Sur ces figures, le vent de mousson crée une situation de $\mathrm{V}$ responsable des précipitations. Lorsque nous avons une dépression à la surface, il y a convergence des vents vers le centre de la dépression. Cela produit un mouvement d'air vers le haut puisque l'air ne peut entrer dans le sol. L'air en montant se refroidit et éventuellement la vapeur d'eau qu'il contient va se condenser et donner lieu à la formation de nuages et surtout de précipitations intenses.

La période juin, juillet, août (JJA) est celle où les inondations sont les plus probables. La figure 9 présente les anomalies journalières de quelques grandeurs météorologiques à Douala entre 1985 et 2019. Il nous permet d'observer les jours où il y a plus de précipitations, de ruissellements, d'évapotranspiration ou de température, les maximums de précipitations

Tableau 1. Les niveaux de retour $(\mathrm{mm})$ de diverses fréquences à Douala durant la période JJA entre 1985 et 2019 .

Périodes de retour (ans)

\begin{tabular}{lcccccc} 
& $\mathbf{1}$ & $\mathbf{5}$ & $\mathbf{1 0}$ & $\mathbf{2 0}$ & $\mathbf{5 0}$ & $\mathbf{1 0 0}$ \\
\hline Niveaux de retour & 257 & 357 & 465 & 569 & 575 & 631 \\
\hline Intervalle de confiance & {$[230-283$} & {$[333-382]$} & {$[404-527]$} & {$[505-633]$} & {$[507-644]$} & {$[560-702]$} \\
\hline
\end{tabular}

d'intensité bien marquées. Les pluies de mousson sont en général observées entre août et novembre et les pluies de perturbation au début et à la fin de la saison des pluies. Elles sont dues à des systèmes précipitants en $\mathrm{V}$ (Lenouo et al., 2009).

L'analyse fréquentielle des précipitations journalières est donnée dans le tableau 1. Les niveaux de retour (NR) en $\mathrm{mm}$ des précipitations extrêmes à Douala pour les différentes périodes de retour (PR) sont obtenus en considérant les précipitations maximales durant la période JJA entre 1985-2019 (figure 9a). Les valeurs qui représentent l'intervalle de confiance à $95 \%$ associé au NR sont aussi données dans ce tableau. Pour mémoire, si une accumulation sur 24 heures de $73 \mathrm{~mm}$ est une pluie de période de retour 10 ans (ou décennale), c'est que cette pluie se produit statistiquement à la fréquence d'une fois tous les dix ans. La longueur des PR associées aux quantités de précipitations de ces années dépend de leurs intensités et varie d'une zone de la ville de Douala à une autre. Par conséquent, une recherche portant sur la modélisation des événements hydroclimatiques nécessiterait au préalable la prise en compte de l'hétérogénéité spatiale de la distribution des précipitations et devrait aussi reposer sur la topographie. 
(a) $2 \mathrm{hr} \mathrm{HOO}$
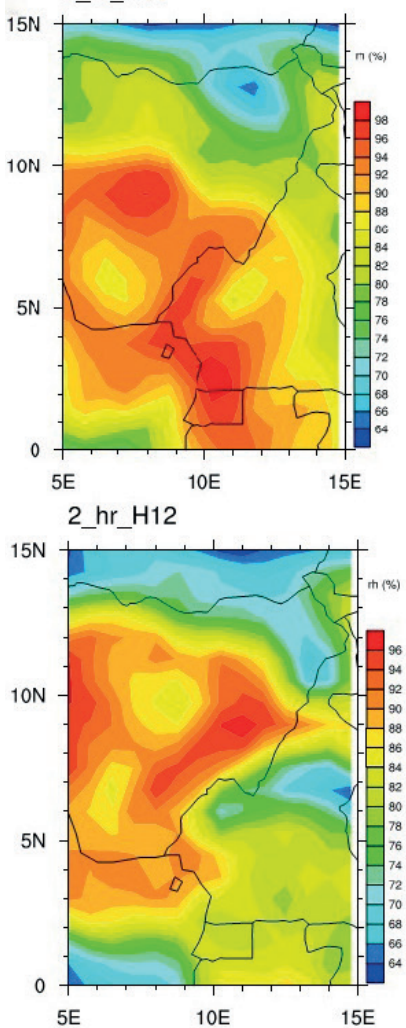

(b) div_00h_2
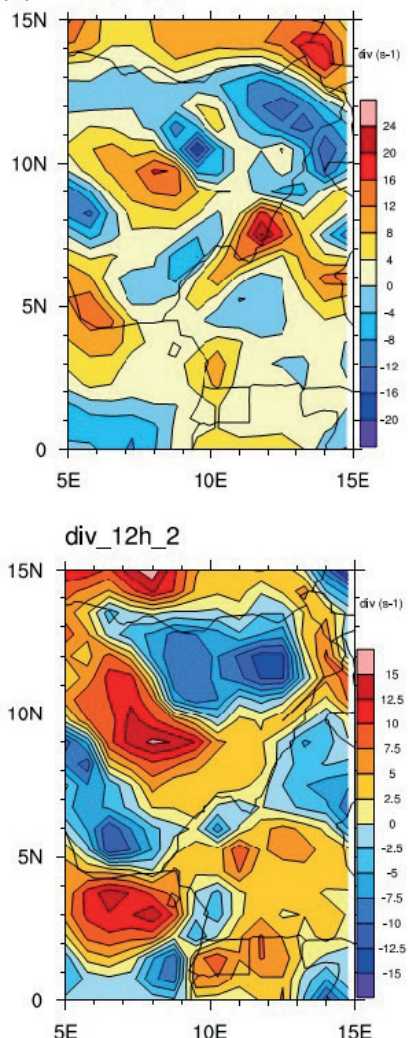

$2 \mathrm{hr} \mathrm{H} 06$

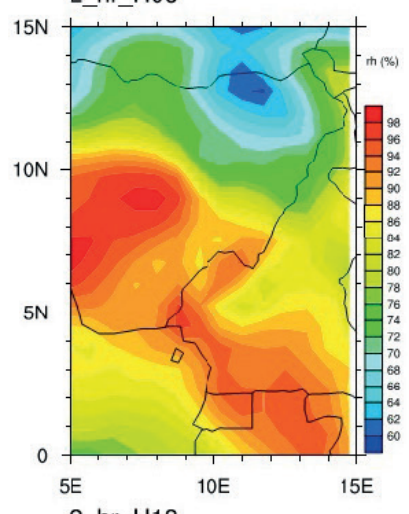

2_hr_H18

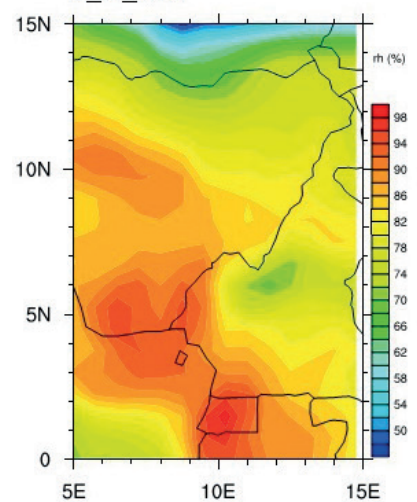

div_06h_2
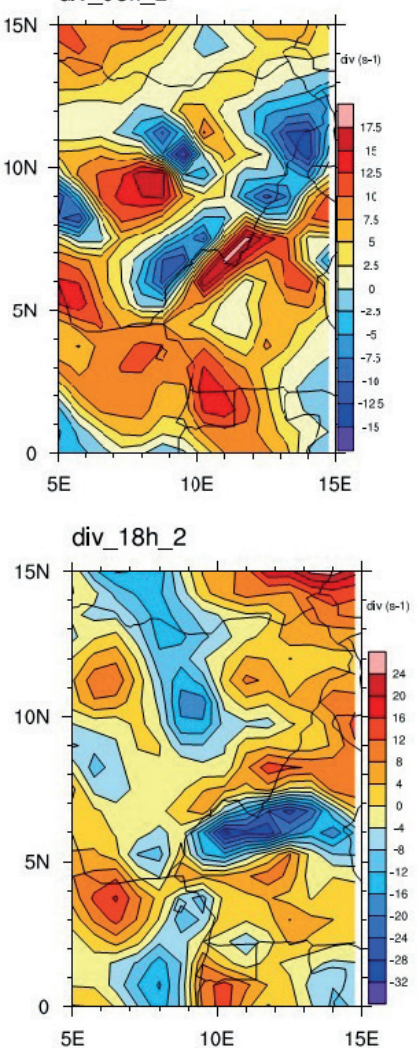

3_hr_H0O

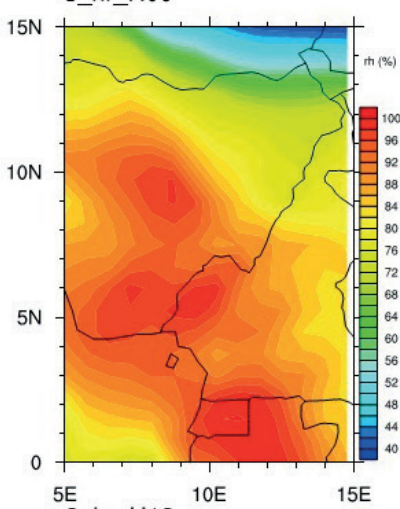

$15 \mathrm{~N}$

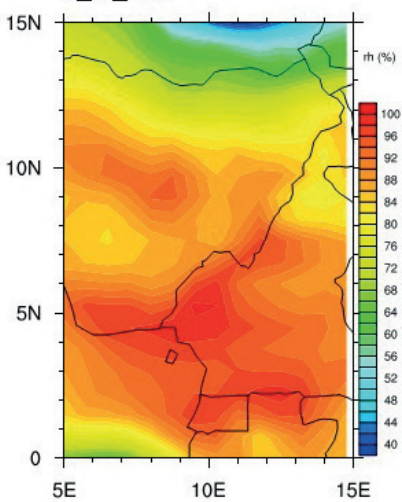

div_00h_3
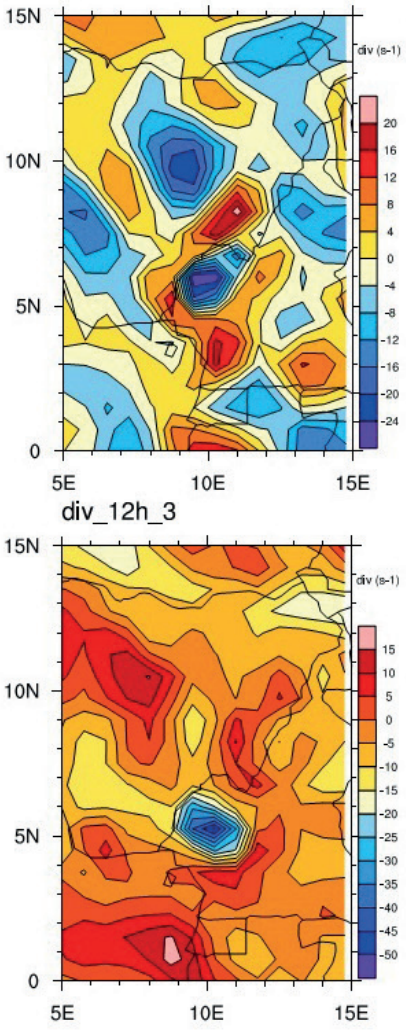

3_hr_H06
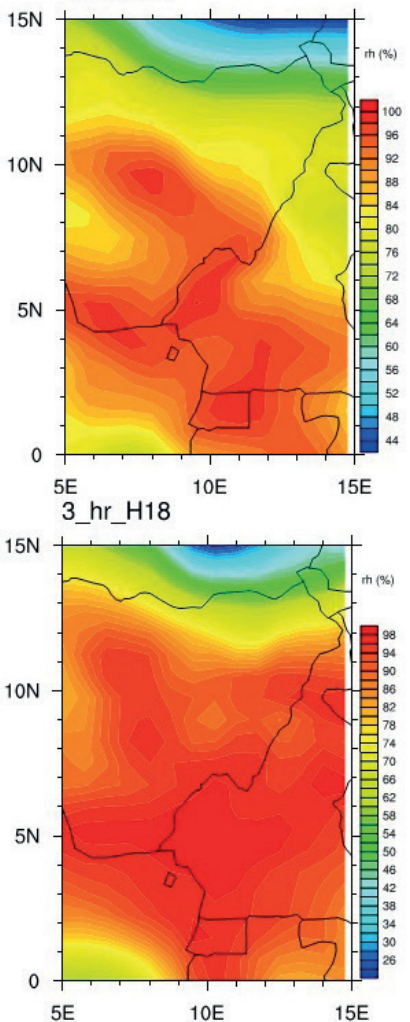

div_06h_3
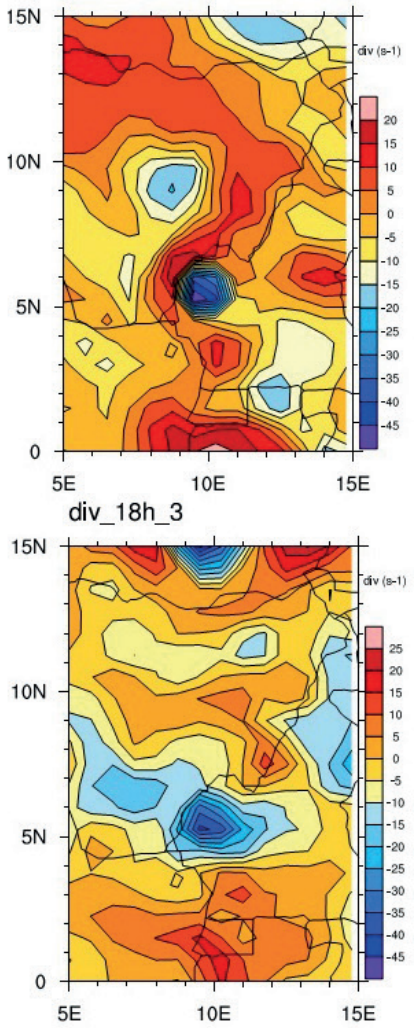

Figure 8. Variation horizontale de (a) l'humidité relative et de (b) la divergence du vent à $850 \mathrm{hPa}$ le 2 et 3 août 2000.

\section{Conclusion}

Nous pouvons conclure que la variabilité météorologique a un impact fort sur les ruissellements à Douala. Cela favorise également les inondations dans la ville de Douala à cause de la géomorphologie de son sol et de sa position au-dessus de la mer. Le ministère de l'administration territoriale (Intérieur) et la communauté urbaine de Douala (CUD) détiennent la responsabilité de la prévision des crues ; les météorologistes et les hydrologues, malgré leur faible effectif, se sont fortement impliqués lors de l'événement.
Ils pourront en permanence préparer des prévisions, des alertes et des rapports pour les organismes de décision et pour le public. Dans l'ensemble, le dispositif de protection contre les inondations et le système de secours ne fonctionnent pas très bien et de nombreuses mesures préventives n'ont pas 

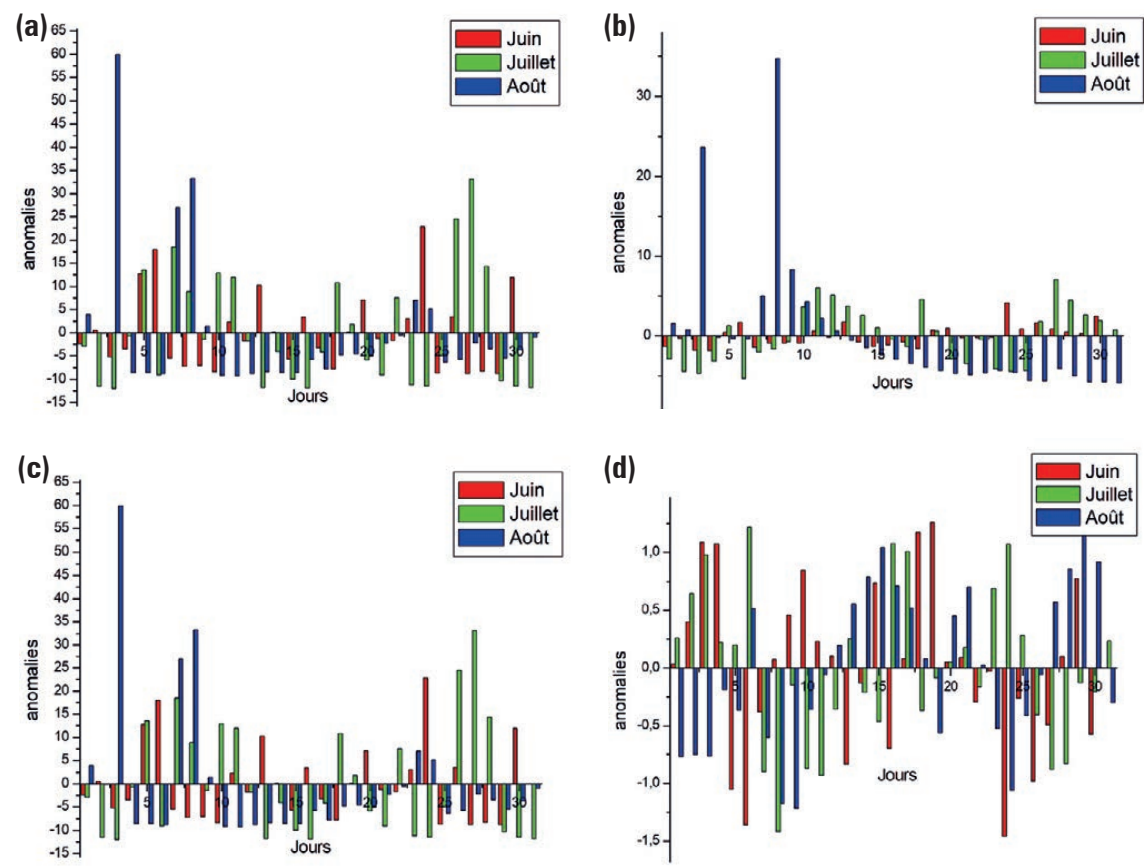

Figure 9. Évolution des anomalies (a) des précipitations, (b) des ruissellements, (c) des évapotranspirations et (d) de la température à 2 mètres à Douala durant la période JJA, moyennées entre 1985 et 2019.

été appliquées pendant la crue, notamment l'évacuation des personnes. De façon générale, le dispositif n'a pas été plus efficace que lors des précédentes inondations catastrophiques à Douala. L'expérience acquise et l'adoption de nouvelles lois concernant la gestion des crises et le système intégré de secours auront à l'avenir un impact très positif en favorisant une conduite rapide et adaptée chez nos concitoyens.
Sur le plan national, le service de la prévention des catastrophes d'origine météorologique de la DMN publie des bulletins qui déterminent l'intensité des impacts dus aux cumuls pluviométriques et la probabilité d'occurrence des événements pluvieux. La carte est établie selon les directives de l'OMM (No. 1150) sur les prévisions et alertes basées sur les impacts multirisques. Ainsi, il est fortement recommandé à l'État du Cameroun de mettre en place un système d'alerte précoce. Cette alerte permettra de prévenir les autorités et le public d'un niveau de risque de crue dans les 24 heures à venir. Elle peut être coordonnée avec le dispositif de vigilance météorologique mis en place par la Direction de la Météorologie Nationale, et qui couvre l'ensemble des risques d'origine météorologique, en les localisant par département. Cela permettra aux autorités locales, notamment les préfets et les maires, ainsi qu'au public, de se mettre en situation de réagir de manière appropriée et de gérer la situation dans de meilleures conditions.

\section{Remerciements}

Ce travail a été fait avec le soutien scientifique, technique et financier du projet PRInce (Programme de recherche interdisciplinaire sur le climat et l'environnement urbain), dans le cadre du projet Douala Ville Durable : développement durable et réhabilitation du site "Maképé Missoké », cofinancé par l'AFD, la FFEM et la CUD. Les auteurs expriment leur gratitude au CEPMMT pour avoir rendu disponibles les données Era-Interim et ERA5. Nous remercions également Meteoblue pour avoir mis à notre disposition les données observées ; ainsi que les éditeurs $\mathrm{O}$. Boucher et M. Zelvelder pour leurs remarques constructives.

\section{Bibliographie}

Amanejieu A., 2019. Analyse temporelle de la représentation du risque d'inondation de 1980 à 2018 à Douala, Cameroun. Mémoire de master, Université de Liège, 167 p., http://hdl.handle.net/2268.2/5575

Bertoni J.C., 2006. Inondations urbaines en Amérique latine : réflexions sur le rôle des facteurs de risque. Frontiers in Flood Research, 305, 1-19

Bruckmann L., Amanejieu A., Zogning M.O., Moffo, Oze P., 2019. Analyse géohistorique de l'évolution spatio-temporelle du risque d'inondation et de sa gestion dans la zone urbaine de Douala (Cameroun). Géographie physique et environnement, 13, 91-113. doi: 10.4000/physio-geo.8038

Cooley D., 2013. Return periods and return levels under climate change. Extremes in a changing climate, 97-114. doi: 10.1007/978-94-007-4479-0 4

De Groeve T., Kugler Z., Brakenridge G.R., 2006. Alerte aux inondations en temps quasi réel pour le système mondial d'alerte et de coordination en cas de catastrophe. Actes ISCRAM2007, 3339.

Fouquet P. et al., 1978. Évacuation des eaux pluviales urbaines. Rapport de l'Amicale des élèves de l'ENPC, Paris, 166 p.

Giec, 2007. Rapport de synthèse. Bilan 2007 des changements climatiques : Conséquences, adaptation et vulnérabilité (Parry M., Canziani 0., Palutikof J., van der Linden P., Hanson C., eds). p. 114. https://www.ipcc.ch/site/assets/uploads/2018/03/ar4-wg2-spm-fr.pdf

Jin M.L., Shepherd J.M., 2005. Inclusion of urban landscape in a climate model: how can satellite data help? Bull. Am. Meteorol. Soc., 86, 681-689. doi: 10.1175/BAMS-86$5-681$

Jones P.D., Groisman P.Y., Coughlan M., Plummer N., Wang W.C., Karl T.R., 1990. Assessment of urbanization effects in time series of surface air temperature over land. Nature, 347, 169-172

Jung H.C., Getirana A., Policelli F., McNally A., Arsenault K.R., Kumar S., Tadesse T., Peters-Lidard C.D., 2017. Upper Blue Nile basin water budget from a multi-model perspective. J. Hydrol., 555, 535-546. doi: 10.1016/j.jhydrol.2017.10.040

Khosla A.N., 1949. Appraisal of water resources (analysis and utilization of data). Proceedings of Unesco conference on the conservation and utilization of resources, Lake Success, New York, 17 
Lenouo A., Monkam D., Vondou D.A., Tanessong R.S., Mkankam K.F., 2009. Analyse des conditions météorologiques pour la sécurité aérienne à Douala. La Météorologie, 65, 46-58.

Masih I., Shreedhar M., Mussá F., Trambauer P., 2014. A review of droughts on the African continent: A geospatial and long-term perspective. Hydrol. Earth Syst. Sci., 18, 3635-3649. doi: 10.5194/hess-18-3635-2014

Meylan P., Favre A.C., Musy A., 2008. Hydrologie fréquentielle : une science prédictive. Lausanne, Presses polytechniques et universitaires romandes, 174 p.

Monfet J., 1979. Évaluation du coeffcient de ruissellement à l'aide de la méthode SCS modifée. Bibliothèque nationale du Québec, ISBN 2-551-03851-0, p. 39.

Mulvaney T.J., 1851. The use of self registring rain and flood gauges in making observations of the relations of rainfall and flood discharges in the given catchment. Proceedings of the Institution of Civil Engineers of Ireland, 4, 19-31.

Paprotny D., Sebastian A., Morales-Napoles 0., 2018. Trends in flood losses in Europe over the past 150 years. Nat. Commun., 9, 1985.

Sighomnou D., Nkamdjou S., Tanyileke G., 1993. Les fortes pluies de la région du Mont-Cameroum : le cas d'Idenau. La Météorologie, 8, 41-47.

Simmons A., Uppala S., Dee D., Kobayashi S., 2006. ERA-Interim: New ECMWF reanalysis products from 1989 onwards. ECMWF Newsletter, 110, 25-35.

Tanessong R.S., Vondou D.A., Djomou Z.Y., Moudi-lgrid P., 2017. WRF high resolution simulation of an extreme rainfall event over Douala (Cameroon): A case study. Modelling Earth Systems and Environment, 3, 927-942.

Tsalefac M., Ngoufo R., Nkwambi W., Djoumessi Tatsngue E., Lengue Fobissie B., 2003. Fréquence et quantité des précipitations journalières sur le territoire camerounais. Publication de I'Association Internationale de Climatologie, 15, 359-367.

Uppala S. et al., 2005. The ERA-40 re-analysis. Q. J. R. Meteorol. Soc., 131, 2961-3012. doi: 10.1256/qj.04.176

Wallez L., 2010. Inondations dans les villes d'Afrique de I'Ouest : diagnostic et éléments de renforcement des capacités d'adaptation dans le Grand Cotonou. Master, université de Sherbrooke, Québec, Canada, $76 \mathrm{p}$.

Wandjie B.B.S., Lenouo A., Monkam D., 2020. Impact of potential evapotranspiration on maize yields in Northern Cameroon using AquaCrop model. Int. J. Hydrol. Sci. Technol., 10, 17-37. doi: 10.1504/IJHST.2020.104984

Weerasinghe I., Bastiaanssen W., Mul M., Jia L., van Griensven A., 2020. Can we trust remote sensing evapotranspiration products over Africa? Hydrol. Earth Syst. Sci., 24, 1565-1586. doi: 10.5194/hess-24-1565-2020

Zizkova E., 2004. Les inondations catastrophiques d’août 2002 en République tchèque. La Météorologie, 46, 34-36. doi: 10.4267/2042/36058 